\title{
What Characterizes Persons Who Do Not Report Musculoskeletal Pain? Results from a 4-year Population-based Longitudinal Study (The Epifund Study)
}

\author{
ELIZABETH A. JONES, JOHN McBETH, BARBARA NICHOLL, RICHARD K. MORRISS, CHRIS DICKENS, \\ GARETH T. JONES, and GARY J. MACFARLANE
}

\begin{abstract}
Objective. To identify and characterize persons in the population who do not report musculoskeletal pain.

Methods. This was a population-based 4-year prospective longitudinal study by postal questionnaire. Population sample recruited from general practice registers in North-West England followed up at 15 months and 4 years.

Results. Of respondents, $17.4 \%$ [95\% confidence interval (CI) $16.1 \%-19.7 \%$ ] reported no pain in the previous month at all 3 measurement intervals over 4 years. They were characterized by low levels of psychological distress [relative risk (RR) low vs high levels of psychological distress 2.3 ; $95 \% \mathrm{CI}$ 1.7-2.9], low levels of depression (2.7; 95\% CI 2.0-3.6), low levels of anxiety (2.1; 95\% CI 1.6-2.7), low health anxiety (1.6; 95\% CI 1.2-2.1), and low illness behavior scores (5.8; 95\% CI 4.0-8.3), good quality sleep (3.4; 95\% CI 2.6-4.4), no somatic symptoms (RR 0 vs 3 or more, 3.1 ; $95 \%$ CI 1.6-6.3) and no adverse life events in the 6 months prior to baseline data collection (RR 0 vs 3 or more, 3.2; 95\% CI 1.6-6.2). On multivariable analysis, good quality sleep, low illness behavior, low psychological distress, and absence of recent adverse life events remained statistically independent predictors of musculoskeletal health. In total, $46 \%$ of persons who had all 4 of these characteristics consistently reported being free of pain, compared to only $5 \%$ of those who had none.

Conclusion. In a general population sample, over a period of 4 years, only around 1 in 6 persons do not report musculoskeletal pain. These persons report low levels of psychological distress and high quality sleep, both of which are potentially modifiable risk factors for the targeting of interventional or preventive strategies. (First Release April 15 2009; J Rheumatol 2009;36:1071-7; doi:10.3899/ jrheum.080541)
\end{abstract}

\section{Key Indexing Terms: \\ MUSCULOSKELETAL PAIN}

Chronic and disabling musculoskeletal pain is common. Pain conditions are considered by many to be typical of the human condition, such that they are largely inescapable ${ }^{1}$. The lifetime prevalence of musculoskeletal pain is reported

From the Aberdeen Pain Research Collaboration (Epidemiology Group), University of Aberdeen, Aberdeen; Arthritis Research Campaign Epidemiology Unit, and Department of Psychiatry, The University of Manchester, Manchester; and School of Community Health Sciences, University of Nottingham, Nottingham, UK.

Supported by the Arthritis Research Campaign, Chesterfield, United Kingdom (grant number 17552).

E.A. Jones, MSc, Aberdeen Pain Research Collaboration (Epidemiology Group), University of Aberdeen; J. McBeth, PhD; B. Nicholl, Arthritis Research Campaign Epidemiology Unit, The University of Manchester; R.K. Morriss, MD, School of Community Health Sciences, University of Nottingham; C. Dickens, MD, Department of Psychiatry, The University of Manchester; G.T. Jones, PhD; G.J. Macfarlane, MD, Aberdeen Pain Research Collaboration (Epidemiology Group), University of Aberdeen.

Address reprint requests to E. Jones, Epidemiology Group, Section of Population Health, University of Aberdeen, School of Medicine and Dentistry, Polwarth Building, Foresterhill, Aberdeen, AB25 2ZD, UK. E-mail: elizabeth.a.jones@abdn.ac.uk

Accepted for publication December 9, 2008.

\section{PATIENT CARE}

\section{EPIDEMIOLOGY}

to be around $65 \%-70 \%$ in the UK and the 1-year prevalence around $30 \%-40 \%{ }^{2}$. Chronic musculoskeletal pain (lasting 3 months or more) is reported by $35 \%-50 \%$ of the population in Sweden ${ }^{3}$ and the 1-year prevalence in Germany is reported as $59 \%^{3}$. But what about those members of the population who do not report any musculoskeletal pain? What distinguishes those who remain in persistently good musculoskeletal health with respect to pain?

Traditionally, epidemiological studies have examined risk factors for developing specific musculoskeletal pains and this has provided some insight into the etiology of pain. However, using this information on etiology, intervention strategies for the management of common pain conditions in primary care have shown only modest improvements in outcome ${ }^{4}$. If people in good musculoskeletal health are in fact the special cases, what can we learn from them to inform prevention and management strategies for musculoskeletal pain?

If there are common aspects to the etiology of regional and widespread musculoskeletal pains, and there is some 
evidence for this ${ }^{5}$, the risk factors for remaining pain-free will be the inverse of those for developing either regional or widespread musculoskeletal pain. To our knowledge, however, how these risk factors relate to musculoskeletal health has not been previously investigated. The factors that do relate to regional musculoskeletal pains generally, rather than specific pains, will demonstrate the strongest relationships with musculoskeletal health, and these factors, if modifiable, are likely to be those important at a population level. Psychological factors, such as depression and anxiety, somatization and sleep disturbance, traumatic experiences, and an individual's attitudes and beliefs have been associated with musculoskeletal pains at several sites $1,3,6$. We therefore hypothesize that individuals in good musculoskeletal health will demonstrate lower levels of factors such as depression, anxiety, sleep disturbance, traumatic experiences, and somatization.

An alternative approach, therefore, is to identify factors associated with musculoskeletal health (or remaining free of pain). If these factors are modifiable, then they may emphasize important targets for future interventions among persons consulting primary care with musculoskeletal pain, to improve outcome and health promotion efforts to maintain musculoskeletal health.

The objectives of our study were therefore to identify persons in persistently good musculoskeletal health (demonstrated by reporting no pain at repeated measurements) in the population and to attempt to characterize those persons.

\section{MATERIALS AND METHODS}

We conducted a 4-year prospective population-based study of pain in the North-West of England known as the Epidemiology of Functional Disorders (Epifund) study. The baseline survey consisted of a postal questionnaire sent out to 10,987 individuals from general practice registers, as described $^{7}$. In brief, the baseline survey consisted of sampling adults aged between 25 and 65 years from the lists of persons registered with 3 general practices.

Baseline. The baseline survey was performed in 2001, when participants completed questionnaires that asked about pain status using the single question, "During the past month have you had any ache or pain which has lasted for one day or longer?". Psychosocial factors known to be associated with musculoskeletal pain were measured using the instruments described below.

Psychosocial factors. Psychological distress: The 12-item General Health Questionnaire (GHQ) was used to measure psychological distress. Originally designed as a screening test for psychological disorders in the community, items relate to elements including decision-making, concentration, and feelings of depression in the "past few weeks" and are scored present or absent, giving a maximum score of 12 . The GHQ-12, previously used in population surveys as a measure of generalized distress, has been shown to have high sensitivity but low specificity, and it is as effective a measure for detecting cases of distress as the longer GHQ- $28^{8}$.

Depression: The depression subscale of the Hospital Anxiety and Depression Scale (HADS) was used to evaluate depression. It was developed as a self-complete screening test to identify anxiety and depression in non-psychiatric hospital settings but has been extensively used in research both in and out of the clinical setting. The depression subscale consists of 7 items asking about aspects of depression the previous week. Responses are coded from 0 to 3 , giving a total score up to 21, with a high score denoting high likelihood of the presence of depressive disorder ${ }^{9}$.
Anxiety: Two measures of anxiety were used in the Epifund study, the HADS, which measures general anxiety, and the Illness Attitudes Scale (IAS), which contains a subscale measuring anxiety as it pertains to health. The HADS has been shown to be valid and reliable and the 2 subscale dimensions stable across different medical settings and age groups ${ }^{10}$. As with the depression subscale, responses are coded from 0 to 3 , giving a total score up to 21 , with a high score denoting high likelihood of the presence of anxiety 9 . The IAS was developed to measure psychopathology. The health anxiety portion comprises 11 items with Likert scale responses coded $0-4$. This gives a maximum possible anxiety score of 44 . The scale has been validated and is deemed to have good internal consistency ${ }^{11}$.

Illness behavior: The illness behavior subscale of the IAS asks questions about common illness behaviors, including questions on the number of practitioners seen, changes in treatment, and frequency of healthcare consulting in the past year. It comprises 3 items coded in the same way as the health anxiety items and 3 with numerical or frequency answers (for example, number of doctors or healers seen, frequency of seeing a doctor), giving a maximum possible illness behavior score of 24 . The higher the score, the more illness behavior is reported, correlating with poor general health and hypochondriasis ${ }^{11}$.

Sleep problems: The Sleep Problems Scale is a 4-item scale designed to be either used by interviewers or self-administered. Responses are scored $0-5$, giving a maximum total score of 20 . The scale has good test-retest reliability and good internal validity, it is also well tolerated by participants ${ }^{12}$. It has previously been used in studies of insomnia treatments ${ }^{13}$.

Somatization: The Screening Test for Somatisation Disorder (STSD) is a checklist that was developed as a tool for use in screening for somatization disorder, whereby subjects' psychological distress manifests in the form of physical symptoms. The version used in the Epifund questionnaires was modified slightly from the original STSD in that the question "Was there ever a time when you lost your voice for $30 \mathrm{~min}$ or more and couldn't speak above a whisper?" was substituted for the question "Have you ever had burning sensations in your sexual organs, mouth or rectum?". This substitution was made to render the STSD more acceptable to study participants in the general population. Items were scored as present or absent, giving a total score out of 6 for men and 7 for women (for whom there is a question on menstrual cramps) ${ }^{14}$.

Life events: The List of Threatening Experiences is a 12-item checklist scale that includes common life events that are likely to be psychologically threatening. All items are scored as present or absent, giving a total score possible of 12 . The list was developed to provide a brief and simple tool for clinical assessment in social psychiatry. The checklist has good test-retest validity and shows high specificity and sensitivity. The scale has been successfully used both clinically and in research settings ${ }^{15,16}$.

Assessment of musculoskeletal health. Participants were followed up twice by further postal questionnaires at 15 months and 4 years from baseline. In the followup questionnaires, participants were asked the same question about pain in the previous month as had been included in the baseline survey. Persistent pain-free status was used as a measure of musculoskeletal health. Participants were asked at each of the 3 timepoints: During the past month have you had any ache or pain which has lasted for one day or longer? Participants who gave a negative response at all 3 timepoints were defined as in good musculoskeletal health (not reporting any pain) and compared to the rest of the study population, who gave any other combination of responses.

Analysis. All analysis was performed using Stata 10.0 (StataCorp, College Station, TX, USA). Total scores for GHQ, the 2 IAS subscales, the 2 HAD subscales, and Estimation of Sleep Problems were grouped into tertiles: "Low," "Medium," and "High." The List of Threatening Experiences scores were divided into "no events," "one or two events," and "three or more events." The responses to the STSD were grouped into "no symptoms," "one symptom," "two symptoms," and "three or more symptoms." In each case the highest group were used as the referent categories.

Associations between musculoskeletal health and the psychosocial 
domains were examined using Poisson regression with robust estimates of standard error ${ }^{17}$. Relative risks (RR) were adjusted for age and sex and are presented with $95 \%$ confidence intervals (CI). Domains found to be associated with good musculoskeletal health (as marked by repeatedly reporting no pain) were offered to a forward stepwise Poisson regression model to examine their independent contributions to the prediction of good musculoskeletal health.

The internal validity of the analysis was assessed by comparing the age and sex profile of full participants with that of those who participated at baseline. The multivariable model was weighted back to the baseline age and sex profile to ascertain the effect any differences had on the conclusions of the model.

Ethical approval. The project had ethics committee approval from South Manchester Local Research Ethics Committee and South Cheshire Local Research Ethics Committee.

\section{RESULTS}

Participation. Of the 6791 baseline participants, 3985 and 2761 completed first and second followup surveys, respectively, as shown in Figure 1. This gives participation rates of $68 \%$ at 15 months and $52 \%$ at 4 years, adjusted for those who had died or moved away. Of participants with baseline and outcome data on all 3 occasions, 2260 gave full psychosocial data. Participants who responded at all 3 timepoints and gave full outcome data were $41 \%$ male, $59 \%$ female. The mean age of participants was 46.0 years (interquartile range 36.8-55.1 yrs).

Presence of outcome. Four hundred twenty-one (17.3\%)

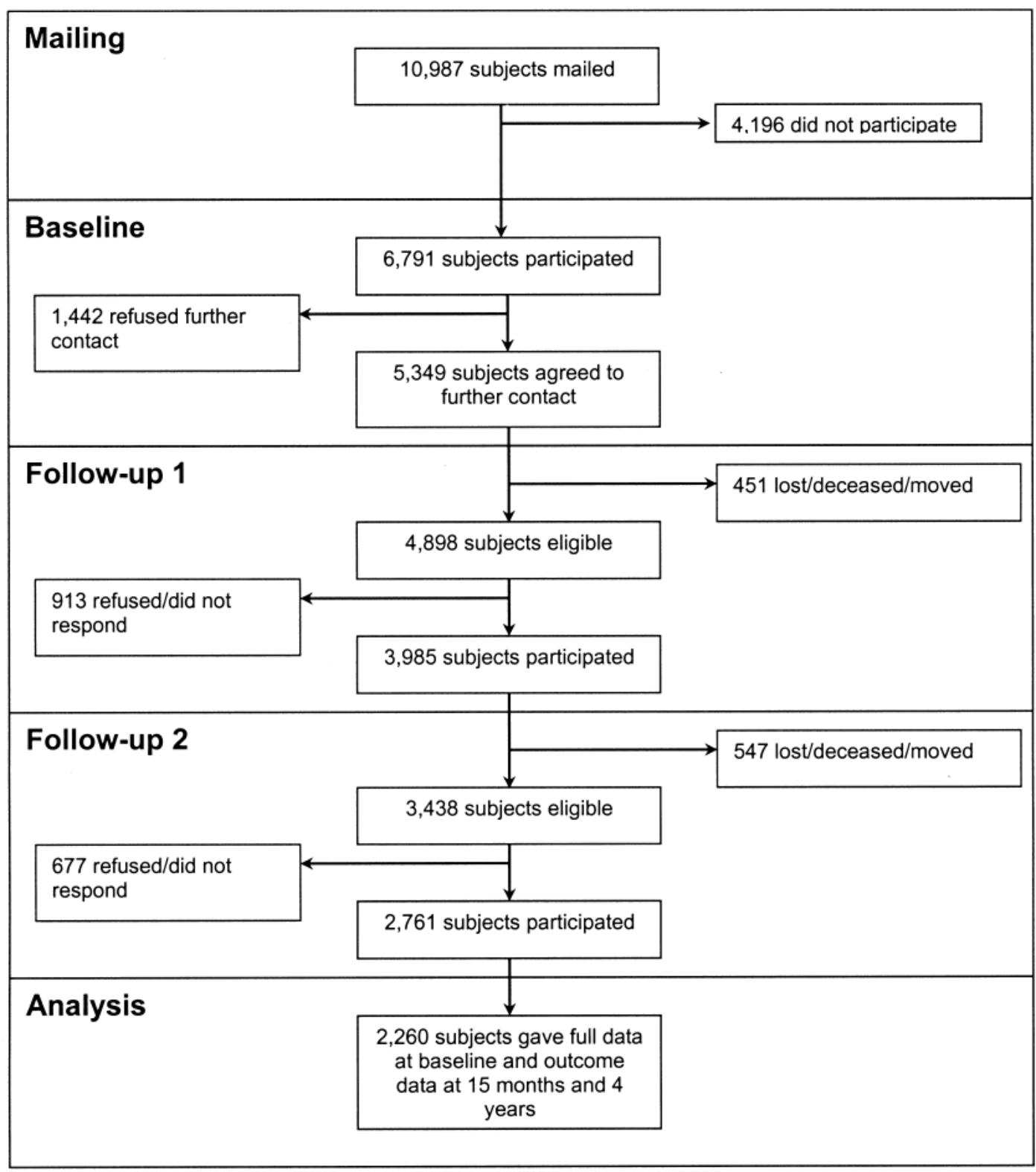

Figure 1. The progress of participants through the study. 
participants did not report pain at any of the 3 data collection points. Persistently reporting good musculoskeletal health was more common in men than women $(20.0 \%$ vs $15.6 \%$; Pearson chi-squared $=7.99, \mathrm{p}=0.005$ ), but there was no significant relationship with age (age defined as a categorical variable with categories 25-35, 36-45, 46-55, and 56-65 yrs; Pearson chi-squared for trend $=4.75, \mathrm{p}=$ $0.191)$.

Univariable analysis. Musculoskeletal health was predicted by low scores on the scales used to measure distress, depression, anxiety, health anxiety, illness behavior, sleep problems, somatization, and life events. These relationships are shown in Table 1. Subjects with low levels of distress as measured by the GHQ were more than twice as likely not to report any pain at all 3 followup points than those with high scores (RR 2.26, 95\% CI 1.75-2.90), and those with low depression scores were almost 3 times more likely to consistently not report pain as those with high scores (RR 2.70, 95\% CI 2.03-3.58). Subjects reporting never having experi- enced somatic symptoms previously were more than 3 times more likely to not report pain at all 3 followup points than those reporting 3 or more previous somatic symptoms (RR 3.16, 95\% CI 1.61-6.20).

Similarly, subjects reporting low levels of anxiety were significantly more likely to report good musculoskeletal health for the duration of followup - whether measured by HADS (RR 2.08, 95\% CI 1.63-2.66) or the Health Anxiety Subscale of the IAS (RR 1.62, 95\% CI 1.28-2.06). Subjects with low scores on the IAS illness behavior subscale were nearly 6 times more likely than those with high scores not to report pain (RR 5.77, 95\% CI 4.01-8.29) and those with few sleep problems were more than 3 times as likely to report good musculoskeletal health than those returning high scores (RR 3.37, 95\% CI 2.60-4.38). Finally, subjects reporting none of the recent adverse life events listed in the Life Events Inventory were more than twice as likely to consistently report experiencing no pain than those reporting more than 3 such events (RR 2.37, 95\% CI 1.58-3.54).

Table 1. Univariable analysis.

\begin{tabular}{|c|c|c|c|}
\hline \multirow[t]{2}{*}{ Variable } & \multicolumn{2}{|c|}{ Good Musculoskeletal Health, n (\%) } & \multirow{2}{*}{$\begin{array}{c}\text { RR (95\% CI) } \\
\text { (adjusted for age and sex) }\end{array}$} \\
\hline & Yes & No & \\
\hline \multicolumn{4}{|c|}{ Distress (GHQ) } \\
\hline Low & $298(23)$ & $1002(77)$ & $2.26(1.75-2.90)$ \\
\hline Medium & $56(12)$ & $408(88)$ & $1.17(0.83-1.65)$ \\
\hline High & $65(10)$ & $578(90)$ & 1 \\
\hline \multicolumn{4}{|c|}{ Depression (HAD) } \\
\hline Low & $269(24)$ & $862(76)$ & $2.70(2.03-3.58)$ \\
\hline Medium & $95(14)$ & $589(86)$ & $1.57(1.13-2.17)$ \\
\hline High & $51(9)$ & $537(91)$ & 1 \\
\hline \multicolumn{4}{|c|}{ Anxiety (HAD) } \\
\hline Low & $223(22)$ & $780(73)$ & $2.08(1.63-2.66)$ \\
\hline Medium & $116(17)$ & $548(83)$ & $1.65(1.26-2.16)$ \\
\hline High & 77 (11) & $656(89)$ & 1 \\
\hline \multicolumn{4}{|c|}{ Health anxiety (IAS) } \\
\hline Low & $170(21)$ & $638(79)$ & $1.62(1.28-2.06)$ \\
\hline Medium & $142(17)$ & $687(83)$ & $1.36(1.06-1.73)$ \\
\hline High & $88(13)$ & $612(87)$ & 1 \\
\hline \multicolumn{4}{|c|}{ Illness behavior (IAS) } \\
\hline Low & $248(27)$ & $656(73)$ & $5.77(4.01-8.29)$ \\
\hline Medium & 135 (17) & $682(83)$ & $3.53(2.42-5.14)$ \\
\hline High & $31(5)$ & $637(95)$ & 1 \\
\hline \multicolumn{4}{|c|}{ Sleep problems } \\
\hline Low & $234(29)$ & $587(72)$ & $3.37(2.6-4.38)$ \\
\hline Medium & $116(15)$ & $653(85)$ & $1.79(1.33-2.38)$ \\
\hline High & $64(8)$ & $708(92)$ & 1 \\
\hline \multicolumn{4}{|c|}{ Somatic Symptoms (STSD) } \\
\hline 0 & $248(23)$ & $851(77)$ & $3.16(1.61-6.20)$ \\
\hline 1 & $72(13)$ & 499 (87) & $1.79(0.89-3.60)$ \\
\hline 2 & $26(11)$ & $210(89)$ & $1.56(0.73-3.32)$ \\
\hline $3+$ & $8(7)$ & $105(93)$ & 1 \\
\hline \multicolumn{4}{|l|}{ Life events } \\
\hline 0 & $223(23)$ & 815 (79) & $2.37(1.58-3.54)$ \\
\hline $1-2$ & 167 (16) & 909 (84) & $1.73(1.15-2.60)$ \\
\hline $3+$ & $24(9)$ & $237(91)$ & 1 \\
\hline
\end{tabular}

RR: relative risk; GHQ: General Health Questionnaire; HAD: Hospital Anxiety and Depression scale; IAS: Illness Attitudes Scale; STSD: Screening Test for Somatisation Disorder. 
Multivariable analysis. On multivariable analysis, high quality sleep, low levels of illness behavior, low levels of psychological distress, and few recent adverse life events at baseline proved to be independent predictors of good musculoskeletal health such that adding further domains to the model did not significantly improve prediction. This model is shown in Table 2 (Model 1).

Nonparticipants. The characteristics of individuals who completed the study questionnaire at all 3 timepoints were different from those who did not. Completers were more likely to be female (59\% of completers vs $56 \%$ of baseline participants) and older (65\% of completers over $45 \mathrm{yrs}$ vs $53 \%$ of baseline participants). While age was not significantly associated with pain status in our analysis, both these factors have previously been associated with musculoskeletal pain, so the multivariable model was weighted back to the age and sex profile of the participants at baseline. As can also be seen in Table 2 (Model 2), once this model had been weighted, the findings remained largely unchanged.

Fit of the model. There was a significant trend such that the greater the number of these factors an individual reported as low at baseline, the more likely they were to report no pain throughout the study (chi-squared $=158.66, \mathrm{p}<0.001$ ); this

Table 2. Multivariable regression model and weighted model $(\mathrm{n}=1881)$.

\begin{tabular}{|c|c|c|}
\hline Variable & $\begin{array}{l}\text { Model } 1 \\
\text { Relative Risk (95\% CI) }\end{array}$ & $\begin{array}{l}\text { Model } 2 \\
\text { Weighted Relative } \\
\text { Risk }(95 \% \mathrm{CI})\end{array}$ \\
\hline \multicolumn{3}{|l|}{ Age } \\
\hline $25-35$ & 1 (referent) & 1 (referent) \\
\hline $36-45$ & $0.83(0.60-1.13)$ & $0.85(0.62-1.16)$ \\
\hline $46-55$ & $0.90(0.66-1.21)$ & $0.91(0.67-1.22)$ \\
\hline $56-65$ & $0.78(0.57-1.06)$ & $0.80(0.58-1.08)$ \\
\hline \multicolumn{3}{|l|}{ Sex } \\
\hline Male & 1 (referent) & 1 (referent) \\
\hline Female & $1.07(0.88-1.29)$ & $1.10(0.90-1.33)$ \\
\hline \multicolumn{3}{|l|}{ Sleep problems } \\
\hline Low & $2.21(1.62-3.01)$ & $2.12(1.55-2.91)$ \\
\hline Medium & $1.35(0.97-1.88)$ & $1.34(0.96-1.87)$ \\
\hline High & 1 (referent) & 1 (referent) \\
\hline \multicolumn{3}{|l|}{ Illness behavior } \\
\hline Low & $3.33(2.16-5.12)$ & $3.14(2.01-4.90)$ \\
\hline Medium & $2.39(1.55-3.67)$ & $2.21(1.41-3.44)$ \\
\hline High & 1 (referent) & 1 (referent) \\
\hline \multicolumn{3}{|c|}{ Psychological distress } \\
\hline Low & $1.09(0.82-1.45)$ & $1.17(0.87-1.56)$ \\
\hline Medium & $0.79(0.54-1.13)$ & $0.88(0.60-1.28)$ \\
\hline High & 1 (referent) & 1 (referent) \\
\hline \multicolumn{3}{|c|}{ Somatic symptoms } \\
\hline None & $1.49(0.76-2.91)$ & $1.42(0.72-2.78)$ \\
\hline One & $1.11(0.55-2.22)$ & $0.98(0.49-1.94)$ \\
\hline Two & $1.25(0.59-2.62)$ & $1.21(0.58-2.53)$ \\
\hline Three or more & 1 (referent) & 1 (referent) \\
\hline \multicolumn{3}{|l|}{ Life events } \\
\hline None & $1.31(0.85-2.00)$ & $1.30(0.83-2.01)$ \\
\hline One or two & $1.07(0.69-1.64)$ & $1.06(0.68-1.66)$ \\
\hline Three or more & 1 (referent) & 1 (referent) \\
\hline
\end{tabular}

is shown in Figure 2. Prevalence of musculoskeletal health (defined as reporting no pain in the previous month at baseline and each of the followup surveys) was $46.4 \%$ in those reporting low levels (or no adverse events or previous somatic symptoms) in all of the 5 factors, but only $4.4 \%$ in those reporting high or medium levels in all 5 factors. This represents more than a 10-fold decrease in likelihood of repeatedly not reporting pain in the latter group.

\section{DISCUSSION}

To our knowledge this is the first study to examine musculoskeletal health using prospective longitudinal data. We have demonstrated that around 1 in 6 of the population reported no pain (and, we assume, were therefore in good musculoskeletal health) over the 4 years of followup. We have further demonstrated that these persons can be characterized as reporting low levels of psychosomatic factors and high quality sleep.

Various issues of methodology are worthy of discussion, namely the effect of differential completion according to age and sex, the nature of the associations between the factors examined and persistent reporting of no pain, and the validity of the outcome measure. First, over the 4 years of the study, the participants who did not complete full questionnaires were more likely to be male and towards the younger end of the age profile. Thus older people and women were overrepresented in the final analysis compared to the baseline study population. For this reason the original analysis was weighted to investigate the effect this had had on the factors identified (since increased reporting of pain is usually found in older people and women). As can be seen in Table 2, the weighting of the model did not have a substantial effect on the outcome of the regression model and does not change the conclusions. Thus, the validity of the model is confirmed despite the potential bias of differential attrition by sex. Secondly, since the outcome measure (pain) was recorded at baseline, with the measurement of the psychosocial variables, a second methodological issue arose, whereby the differences between participants persistently reporting no pain and others could be attributed to the consequences of experiencing pain at baseline: for example, experiencing pain might lead to subjects reporting poor sleep or high distress. In order to investigate this, a further analysis was performed (not presented here) using only data from those individuals who reported being free of pain at baseline and comparing those who reported being free of pain with those who reported any pain at followup. Among these participants, the prevalence of reporting no pain persistently over the course of the study was around $50 \%$. The analysis showed some attenuation of the associations seen in the original analysis, but the magnitude of effect of good quality sleep remained. Nevertheless, even this supplementary analysis also cannot exclude the possibility of pain leading to poor quality sleep rather than vice versa, since 


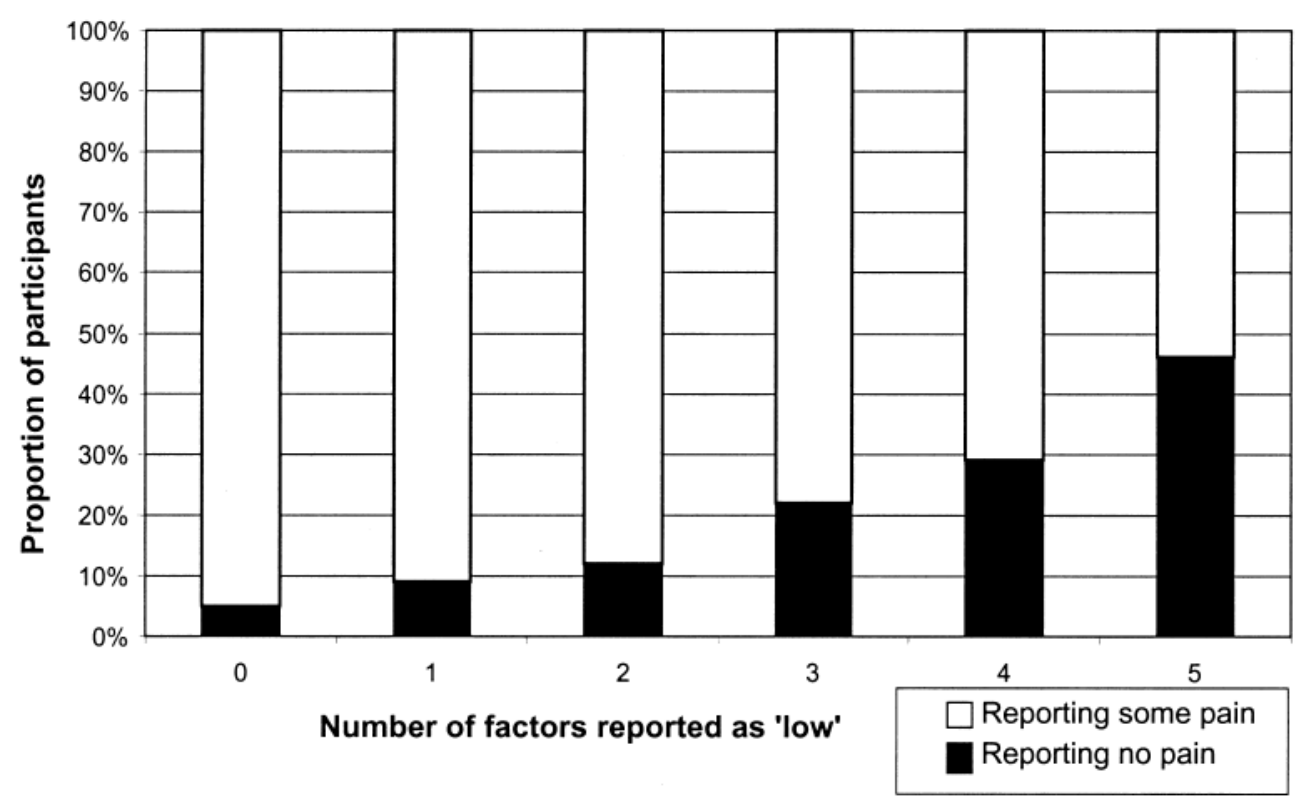

Figure 2. Percentage of participants repeatedly reporting no pain by number of factors reported as low (from sleep problems, illness behavior, distress, somatization, and life events).

people pain-free at baseline may have had previous pain which influenced sleep quality.

A further methodological issue that arose was the definition of the outcome. By asking participants at 3 timepoints over 4 years about pain in the previous month, our definition of musculoskeletal health cannot be sure that participants did not experience any transient pain in the intervening period. This would have the effect of biasing our results towards the null (if participants designated as being in good musculoskeletal health in fact included a number who experienced some pain in between the months that the surveys were concerned with) and the true associations between musculoskeletal health and psychosocial factors would be stronger than we have found.

Our study has confirmed the importance of psychosocial factors for remaining in good musculoskeletal health. We have particularly underlined the importance of low psychological distress, low illness behavior, few somatic symptoms, and good sleep quality. This aligns with previous research in the field of musculoskeletal pain that indicates that depriving healthy subjects of sleep decreases their pain threshold ${ }^{18}$ and that pain severity in patients with fibromyalgia is predicted by previous night sleep quality ${ }^{19}$.

Of the factors identified in the multivariable model clearly, the number of adverse life events a person experiences is not modifiable. This leaves the other domains as potential targets for the improvement of musculoskeletal health and prevention of chronic musculoskeletal pain conditions. In the context of musculoskeletal pain, promoting low illness behavior, low levels of psychological distress, and good quality sleep appear to present themselves as strategies for effective prevention. Each of these factors has been linked with musculoskeletal pain in the past ${ }^{20-22}$ and may be implicated in the etiology of chronic pain conditions. Currently, interventions addressing distress such as Cognitive Behavioral Therapy (CBT) and relaxation techniques are used to treat fibromyalgia ${ }^{23}$, but illness behavior and sleep quality are not routinely addressed. Thus, these factors present potential targets for improving musculoskeletal health and preventing musculoskeletal pain, since both have been shown to be modifiable ${ }^{24,25}$. Sleep interventions may be suitable to add to current pain management strategies, since sleep quality is not typically affected by interventions aimed at improving primary outcomes or pain management ${ }^{26}$, and even when pain or primary outcomes are improved, sleep disturbances may persist or worsen ${ }^{20}$.

The factors we have identified present starting points for the further investigation of musculoskeletal health and how it relates to musculoskeletal pain conditions. Further research is required to establish the effects of musculoskeletal health-promoting interventions on pain conditions whether they can improve pain outcomes, or are better targeted in a preventive fashion - and how it may be appropriate to administer and combine them.

\section{ACKNOWLEDGMENT}

We are grateful for the participation and help of the doctors, staff, and patients of the 3 general practices in Greater Manchester, and Joanne Bradley and Karen Schafheutle for survey administration.

\section{REFERENCES}

1. van Vuuren BJ, van Heerden HJ, Becker PJ, Zinzen E, Meeusen R. Fear-avoidance beliefs and pain coping strategies in relation to 
lower back problems in a South African steel industry. Eur J Pain 2006;10:233-9.

2. Papageorgiou AC, Croft PR, Ferry S, Jayson MI, Silman AJ. Estimating the prevalence of low back pain in the general population. Evidence from the South Manchester Back Pain Survey. Spine 1995;20:1889-94.

3. Bergman S. Public health perspective - how to improve the musculoskeletal health of the population. Best Pract Res Clin Rheumatol 2007;21:191-204.

4. Bogduk N. Management of chronic low back pain. Med J Aust 2004;180:79-83.

5. Macfarlane GJ, Jones GT, McBeth J. Epidemiology of pain. In: McMahon S, Koltzenburg M, editors. Wall and Melzack's textbook of pain. Edinburgh: Churchill Livingstone; 2005: ch. 76.

6. Diamond S, Borenstein D. Chronic low back pain in a working-age adult. Best Pract Res Clin Rheumatol 2006;20:707-20.

7. Gupta A, Silman AJ, Ray D, et al. The role of psychosocial factors in predicting the onset of chronic widespread pain: results from a prospective population-based study. Rheumatology 2007; $46: 666-71$.

8. Goldberg DP, Gater R, Sartorius N, et al. The validity of two versions of the GHQ in the WHO study of mental illness in general health care. Psychol Med 1997;27:191-7.

9. Zigmond AS, Snaith RP. The Hospital Anxiety and Depression Scale. Acta Psychiatr Scand 1983;67:361-70.

10. Spinhoven P, Ormel J, Sloekers PPA, Kempen GIJM, Speckens AEM, van Hemert AM. A validation study of the Hospital Anxiety and Depression Scale (HADS) in different groups of Dutch subjects. Psychol Med 1997;27:363-70.

11. Speckens AEM, Spinhoven P, Sloekers PPA, Bolk JH, van Hemert AM. A validation study of the Whitely Index, the Illness Attitudes Scales, and the Somatosensory Amplification Scale in general medical and general practice patients. J Psychosom Res 1996;40:95-104.

12. Jenkins CD, Stanton BA, Niemcryk SJ, Rose RM. A scale for the estimation of sleep problems in clinical research. J Clin Epidemiol 1988;41:313-21.

13. Morin CM. Measuring outcomes in randomised clinical trials of insomnia treatments. Sleep Med Rev 2003;7:263-79.
14. Othmer E, DeSouza C. A screening test for somatization disorder (hysteria). Am J Psychiatry 1985;142:1146-9.

15. Brugha T, Bebbington P, Tennant C, Hurry J. The List of Threatening Experiences: a subset of 12 life event categories with considerable long-term contextual threat. Psychol Med 1985;15:189-94.

16. Brugha TS, Cragg D. The List of Threatening Experiences: the reliability and validity of a brief life events questionnaire. Acta Psychiatr Scand 1990;82:77-81.

17. Greenland S. Model-based estimation of relative risks and other epidemiologic measures in studies of common outcomes and in case-control studies. Am J Epidemiol 2004;160:301-5.

18. Lautenbacher S, Kundermann B, Krieg J-C. Sleep deprivation and pain perception. Sleep Med Rev 2006;10;357-69.

19. Bigatti SM, Hernandez AM, Cronan TA, Rand KI. Sleep disturbances in fibromyalgia syndrome: Relationship to pain and depression. Arthritis Care Res 2008;59:961-7.

20. Moldofsky H. Sleep and pain. Sleep Med Rev 2001;5:387-98.

21. Macfarlane GJ, Croft PR, Schollum J, Silman AJ. Widespread pain: is an improved classification possible? J Rheumatol 1996;23:1628-32.

22. Hunt IM, Silman AJ, Benjamin S, McBeth J, Macfarlane GJ. The prevalence and associated features of chronic widespread pain in the community using the 'Manchester' definition of chronic widespread pain. Rheumatology 1999;38:275-9.

23. Bennett R, Nelson D. Cognitive behavioral therapy for fibromyalgia. Nat Clin Pract Rheumatol 2006;2:416-24.

24. Dickinson WP, Dickinson LM, deGruy FV, Main DS, Candib LM, Rost K. A randomized clinical trial of a care recommendation letter intervention for somatization in primary care. Ann Fam Med 2003; 1:228-35.

25. Morin CM, Hauri PJ, Espie CA, Spielman AJ, Buysse DJ, Bootzin RR. Nonpharmacologic treatment of chronic insomnia. Sleep 1999;22:1134-56.

26. Buenaver LF, Smith MT. Sleep in rheumatic diseases and other painful conditions. Curr Treat Options Neurol 2007;9:325-36. 